\title{
Characterization of immune cell subtypes in three commonly used mouse strains reveals gender and strain-specific variations
}

\author{
Jonathan A. Hensel ${ }^{1} \cdot$ Vinayak Khattar $^{1} \cdot$ Reading Ashton $^{1} \cdot$ Selvarangan Ponnazhagan ${ }^{1}$
}

Received: 3 May 2018 / Revised: 22 August 2018 / Accepted: 12 September 2018 / Published online: 23 October 2018

(c) United States \& Canadian Academy of Pathology 2018

\begin{abstract}
The lack of consensus on bone marrow (BM) and splenic immune cell profiles in preclinical mouse strains complicates comparative analysis across different studies. Although studies have documented relative distribution of immune cells from peripheral blood in mice, similar studies for BM and spleen from naïve mice are lacking. In an effort to establish strainand gender-specific benchmarks for distribution of various immune cell subtypes in these organs, we performed immunophenotypic analysis of BM cells and splenocytes from both genders of three commonly used murine strains (C57BL/6NCr, 129/SvHsd, and BALB/cAnNCr). Total neutrophils and splenic macrophages were significantly higher in C57BL/6NCr, whereas total B cells were lower. Within C57BL/6NCr female mice, BM B cells were elevated with respect to the males whereas splenic mDCs and splenic neutrophils were reduced. Within BALB/cAnNCr male mice, BM CD4 ${ }^{+}$Tregs were elevated with respect to the other strains. Furthermore, in male BALB/cAnNCr mice, NK cells were elevated with respect to the other strains in both $\mathrm{BM}$ and spleen. Splenic $\mathrm{CD} 4^{+}$Tregs and splenic $\mathrm{CD} 8^{+} \mathrm{T}$ cells were reduced in male BALB/c mice in comparison to female mice. Bone marrow $\mathrm{CD} 4^{+} \mathrm{T}$ cells and $\mathrm{mDCs}$ were significantly increased in $129 / \mathrm{SvHsd}$ whereas splenic CD8 ${ }^{+} \mathrm{T}$ cells were reduced. In general, males exhibited higher immature myeloid cells, macrophages, and NK cells. To our knowledge, this study provides a first attempt to systematically establish organ-specific benchmarks on immune cells in studies involving these mouse strains.
\end{abstract}

\section{Introduction}

Hematopoiesis is a tightly regulated biological process $[1$, 2]. The immune cells in hematopoietic organs undergo constant differentiation, proliferation, and programmed cell death [3-5]. This in turn orchestrates a finely regulated immune cell homeostasis, ensuring that leukocyte subpopulations are maintained within a constant range [3-5]. In mice, the bone marrow (BM) and spleen are two major hematopoietic organs [5, 6]. Although various immune

These authors contributed equally: Jonathan A. Hensel, Vinayak Khattar

Electronic supplementary material The online version of this article (https://doi.org/10.1038/s41374-018-0137-1) contains supplementary material, which is available to authorized users.

Selvarangan Ponnazhagan

sponnazh@uabmc.edu

1 Department of Pathology, University of Alabama at Birmingham, Birmingham, AL 35294, USA cell subsets from these organs are commonly evaluated in studies, there is a lack of strain- and gender-specific benchmarks for each leukocyte cell subtype for these organs in healthy mice [7-9].

In contrast to studies on peripheral blood, where the frequency of leukocyte subpopulations have been charted out in clear detail, there is a gap regarding numerical range for immune cell subtypes in adult murine hematopoietic organs [10-16]. The recent success of cancer immunotherapy has fueled an explosive rise in preclinical translational studies to develop new treatment strategies [17]. However, many such studies are designed to focus on the biology of only specific leukocyte subpopulations in response to treatments with experimental agents [7, 18-23]. Based on the experimental design of the immunological study, in vivo experiments are carried out in different mouse strains and immune profiles are evaluated at different steps following treatment regimen [24]. The variations in experimental variables such as mouse strain, animal physiology, age, gender, drug combinations, time-points, dose, treatment strategies, tumor subtypes, and tumor inoculation methods can create infinite confounders that influence the immune 
parameters and need to be considered even for a study with a single agent $[7,9-15,24-30]$.

For example, Petkova et al. [15] have reported marked differences in the relative proportions of leukocyte subpopulations in peripheral blood among different mouse strains. The SJL/J strains exhibit inverse B and $\mathrm{T}$ cell ratios in comparison to the $\mathrm{C} 57 \mathrm{BL} / 6 \mathrm{NCr}$ mice, highlighting the effect of strain on general immune profile of the organism [9]. Gajewski et al. [28] found that mice from The Jackson Laboratory (JAX) exhibited a dramatically different immune response to implanted melanoma tumors in comparison to mice from Taconic Biosciences (TAC). Similarly, gender also impacts Th1 cells, Tregs, and DCs in both intestinal and peripheral immune populations and has been suggested to be an underlying cause for susceptibility to intestinal disorders [25]. However, there have been no systematic studies to analyze whether such strain- and genderspecific differences exist in murine hematopoietic organs. Hence, there is a pressing need for comprehensive studies to address strain- and gender-specific characterization of the entire gamut of leukocyte subpopulations in normal mice $[10,11,31]$. Since the raw flow cytometry data can be normalized differently (e.g. cell number vs. percentage, percentage leukocytes vs. percentage lymphocytes), a cross-comparison of any immune cell subtype among different studies is obscured [32-34]. Therefore, a lack of specific benchmarks on basal immune cell distribution confounds comparative immunophenotypic analysis across different studies and eventually leads to discordant data. In order to determine the distribution of individual immune cell subtypes in $\mathrm{BM}$ and splenic isolates of three commonly used mouse strains, we conducted an unbiased analysis of cells from $\mathrm{BM}$ and spleen of both genders.

The three major immunocompetent murine strains (C57BL/6NCr, BALB/cAnNCr, 129/SvHsd) analyzed in the present study are broadly used in preclinical research [29, 35]. Whereas BALB/c mice, which are recognized for their use in cancer research, readily develop tumors in response to carcinogenic stimuli, and develop spontaneous tumors at later stages of their lifespan [29, 35-37]; C57BL/6 mice are multifunctional model organisms routinely used in studies involving infectious diseases, congenital anomalies, and cancer $[29,35,38] .129 / \mathrm{Sv}$ mice, on the other hand, are frequently employed in both transgenic/knockout models and oncology studies [39-41]. We evaluated a spectrum of multiple immune cell types representing both the myeloid and lymphoid lineages of hematopoietic cells in these three mouse strains and found important strain- and gender-specific trends within cells of both lineages. Results of the study underscore that both innate and cell-mediated immune profile varies dramatically based on mouse strain and gender.

\section{Methods}

\section{Reagents and antibodies}

RBC lysis buffer was purchased from Bio-Legend (BioLegend, San Diego, CA). FACS buffer was reconstituted by adding $3 \%$ fetal bovine serum (FBS) to ice cold $1 \times$ phosphate-buffered saline (PBS). FBS was purchased from Atlanta Biologicals (Flowery Branch, GA). Antibodies were purchased from BD Biosciences (San Jose, CA), eBioscience (San Diego, CA), Miltenyi Biotec (Auburn, CA), and Thermo-Fischer (Waltham, MA). The clone number and dilution of antibodies are provided in Table 1. The conjugated fluorophores used in the study are enlisted in Table 2. UltraComp ${ }^{\mathrm{TM}}$ eBeads were purchased from eBiosciences and used for compensation. Mouse $F_{c}$ Blocking Reagent was purchased from Miltenyi Biotec. Fixation/Permeabilization Solution Kit with BD GolgiPlug $^{\mathrm{TM}}$ kit and Mouse Foxp3 Buffer Set was used for intracellular staining and purchased from BD Biosciences.

\section{Mouse}

Eight-week-old female and male pathogen-free BALB/ cAnNCr, C57BL/6NCr, and 129/SvHsd mice were obtained from Envigo RMS, Inc. (Indianapolis, IN). Animals were housed in accordance with established guidelines and protocols approved by the UAB Institutional Animal Care and Use Committee (UAB-IACUC).

\section{Isolation of immune cells and flow cytometry}

On the day of the analysis, mice from each strain were first anaesthetized and then sacrificed by cervical dislocation. BM cells were obtained by flushing the marrow cavities with FACS buffer (PBS with 3\% FBS) using an insulin syringe with $28 \mathrm{G}$ needle, followed by passage through a $100 \mathrm{~mm}$ sterile cell strainer. Splenic cells were obtained by gentle pressure-dissociation of spleen using FACS buffer, and then passed through a $100 \mathrm{~mm}$ sterile cell strainer. For all experiments, cells were washed with FACS buffer and pelleted. Red blood cells were lysed by addition of $5 \mathrm{ml}$ RBC lysis buffer (Bio-Legend, San Diego, CA), mixed briefly to re-suspend cells, and incubated for $5 \mathrm{~min}$ at room temperature, followed by FACS buffer wash and spin. Cells were re-suspended in FACS buffer and incubated with $\mathrm{F}_{\mathrm{c}}$ block at room temperature for $15 \mathrm{~min}$. Cells were divided into individual tubes for respective cell type analysis, suspended in $100 \mu \mathrm{l}$ of FACS buffer, and stained for the designated cell type. The data were acquired on BD LSRII (BD Biosciences, New Jersey) and analyzed using FlowJo software (FlowJo LLC, Oregon). 
Table 1 Cell surface markers utilized for characterization of immune cell types

\begin{tabular}{lllll}
\hline Cell type & Markers & & & \\
\hline iMCs & CD11b VioBlue & Gr-1 APC-eFluor780 & $\begin{array}{l}\text { Ly6B }^{-} \text {FITC and F4/80 } \\
\text { Ly6B }{ }^{+/+} \text {FITC and F4/80 APC }\end{array}$ & \\
Macrophages & CD11b VioBlue & F4/80 APC & CD68 PE & \\
mDC & CD11b VioBlue & CD11c PE & Siglec H FITC \\
pDC & CD11b VioBlue & CD11c PE & B220 Vio770 & FoxP3 APC \\
CD4 T cells & CD3 APC-Cy7 & CD4 eFluor450 & & \\
CD4 Tregs & CD3 APC-Cy7 & CD4 eFluor450 & CD25 PE & \\
CD8 T cells & CD3 APC-Cy7 & CD8 FITC & & F4/80 APC \\
B cells & B220 Vio770 & CD19 PE & & \\
NK cells & CD3 APC-Cy7 & NKp46 FITC & & Ly6b FITC \\
Neutrophils & CD11b VioBlue & Gr-1 APC-eFluor780 & LPC \\
\hline
\end{tabular}

${ }^{a}$ Denotes absence of marker for indicated cell type
Table 2 Clone number, dilution, and fluorophores antibodies employed for analysis of immune cell subtypes

\begin{tabular}{lllll}
\hline Antibody & Fluorochrome & Vendor & Clone \# & $\begin{array}{l}\text { Dilution } \\
\text { factor }\end{array}$ \\
\hline CD3e & APC-Cy7 & $\begin{array}{l}\text { BD } \\
\text { Biosciences }\end{array}$ & 145-2C11 & $1: 100$ \\
CD4 & eFluor450 & eBioscience & GK1.5 & $1: 100$ \\
CD8 & FITC & eBioscience & $53-6.7$ & $1: 167$ \\
CD11b & VioBlue & Miltenyi & M1/ & $1: 27$ \\
& & Miltenyi & N418 & $1: 27$ \\
CD11c & PE & Miltenyi & 6D5 & $1: 20$ \\
CD19 & PE & eBioscience & PC61.5 & $1: 200$ \\
CD25 & PE & Miltenyi & FA-11 & $1: 27$ \\
CD68 & PE & Miltenyi & RA3-6B2 & $1: 27$ \\
B220 & APC-Vio770 & Miltenyi & REA126 & $1: 27$ \\
F4/80 & APC & eBioscience & FJK-16s & $1: 20$ \\
FoxP3 & APC & eBioscience & RB6-8C5 & $1: 83$ \\
Gr1 & APC-eFluor780 & Abcam & 7/4 & $1: 154$ \\
Ly6b & FITC & Miltenyi & 29A1.4.9 & $1: 27$ \\
NKp46 & FITC & eBioscience & eBio440c & $1: 200$ \\
Siglec H & FITC & & & \\
\hline
\end{tabular}

\section{Gating strategies}

Markers used for immune cell strategies are described in Tables 1 and 2. The gating strategy for immune cell types is shown in Supplementary Figures 1 and 2. All gating was performed off of live cell populations. Unstained controls and single color controls were utilized to establish baseline gate settings for each respective antibody-fluorophore(s) combination used in individual experiments. The gating strategy for each cell type examined is outlined below and visually represented in Supplementary Figures 1 and 2.

$C D 4^{+} T$ cells: $\mathrm{P} 1=$ live cells, $\mathrm{P} 2=\mathrm{CD} 3 \mathrm{APC}-\mathrm{Cy} 7$, and CD4 eFluor450 double-positive gate.
CD $8^{+} T$ cells: $\mathrm{P} 1=$ live cells, $\mathrm{P} 2=\mathrm{CD} 3 \mathrm{APC}-\mathrm{Cy} 7$ and CD8 FITC double-positive gate.

CD4 ${ }^{+}$Tregs: $\mathrm{P} 1=$ live cells, $\mathrm{P} 2=\mathrm{CD} 3 \mathrm{APC}-\mathrm{Cy} 7$ and CD4 eFluor450 double-positive gate, P3 (intracellular staining $)=\mathrm{CD} 25 \mathrm{PE}$ and FoxP3 APC double-positive gate.

$B$ cells: $\mathrm{P} 1=$ live cells, $\mathrm{P} 2=\mathrm{CD} 19 \mathrm{PE}$, and $\mathrm{B} 220$ Vio770 double-positive gate.

NK cells: $\mathrm{P} 1=$ live cells, $\mathrm{P} 2=\mathrm{CD} 3$ APC-Cy7 negative, and NKp46 FITC positive gate.

Immature myeloid cells (iMCs): $\mathrm{P} 1=$ live cells, $\mathrm{P} 2=$ CD11b VioBlue and Gr-1 APC-eFluor780 double-positive gate, $\mathrm{P} 3=\mathrm{F} / 80$ APC negative and Ly6b FITC negative, F4/80 APC positive and Ly6b FITC negative, F4/80 APC positive and Ly6b FITC positive gate.

Neutrophils: $\mathrm{P} 1=$ live cells, $\mathrm{P} 2=\mathrm{CD} 11 \mathrm{~b}$ VioBlue and Gr-1 APC-eFluor780 double-positive gate, P3 $=$ F4/80 APC negative and Ly6b positive gate.

Macrophages: $\mathrm{P} 1=$ live cells, $\mathrm{P} 2=\mathrm{CD} 11 \mathrm{~b}$ VioBlue and F4/80 APC double-positive gate, P3 = CD68 PE (intracellular staining) positive histogram gate.

$m D C: \mathrm{P} 1=$ live cells, $\mathrm{P} 2=\mathrm{CD} 11 \mathrm{~b}$ VioBlue and CD11c PE double-positive gate.

$p D C: \mathrm{P} 1=$ live cells, $\mathrm{P} 2=\mathrm{CD} 11 \mathrm{~b}$ VioBlue negative and CD11c PE positive gate, $\mathrm{P} 3=\mathrm{B} 220 \mathrm{Vio} 770$ and Siglec $\mathrm{H}$ FITC double-positive gate.

\section{Statistical analysis}

Data were analyzed with multi-variate analysis using twoway ANOVA with multiple comparisons corrected with the Tukey test within Prism ${ }^{\mathrm{TM}}$. Results are represented as scatter plot graphs indicating each group's Mean and SD. Differences were considered significant if $p \leq 0.05$. Sample values at or beyond $\pm(1.7 \times \mathrm{SD})$ were excluded from the analysis. Overall statistics describing the source of variation is listed in Supplementary Figure 3. 


\section{Results}

\section{$\mathrm{CD4}^{+} \mathrm{T}$ cells are significantly elevated in 129 /SvHsd mice}

$\mathrm{CD}^{+}{ }^{+} \mathrm{T}$ cells are critical players in cell-mediated and humoral immunity and differentiate into a specialized $\mathrm{CD} 4^{+}$ subtype based on interactions with antigen-MHC complex and resident cytokine level [42]. The percentages of $\mathrm{CD}^{+}$ $\mathrm{T}$ cells in $\mathrm{BM}$ and spleen ranged from mean values of $3.15 \%$ to $7.76 \%$ and $18.80 \%$ to $31.67 \%$, respectively, in the three murine strains analyzed (Fig. 1a, b). In all cases, CD4 $+\mathrm{T}$ cells were significantly higher in the spleen as compared to the BM (Fig. 1). We observed many strain- and gender-specific variations in $\mathrm{CD} 4^{+} \mathrm{T}$ cells among all three strains. 129/SvHsd mice exhibited a significantly higher BM CD4 ${ }^{+} \mathrm{T}$ cell population in both genders (Fig. 1a, $p \leq 0.001 \delta^{\star}$ and $p \leq 0.0001$ o for C57BL/6NCr; $p \leq 0.0001$ for BALB/cAnNCr). In BM, C57BL/6NCr males exhibited a significantly higher $\mathrm{CD} 4^{+} \mathrm{T}$ cell percentage in comparison to BALB/cAnNCr males (Fig. 1a, $p \leq 0.01$ ). As observed in $\mathrm{BM}$, splenic $\mathrm{CD}^{+} \mathrm{T}$ cells in $129 / \mathrm{SvH}$ sd mice were also significantly elevated with respect to C57BL/6NCr (Fig. 1b, $p \leq 0.00010^{-}, p \leq 0.001$ \%). Splenic $\mathrm{CD}^{+} \mathrm{T}$ cells in male $129 / \mathrm{SvHsd}$ mice were significantly higher in comparison to $\mathrm{BALB} / \mathrm{cAnNCr}$, but this trend was reversed in female mice (Fig. $1 \mathrm{~b}, p \leq 0.05 \delta^{+}$and $p \leq 0.001$ ). Splenic $\mathrm{CD} 4^{+} \mathrm{T}$ cells were significantly elevated in BALB/cAnNCr in comparison to C57BL/6NCr (Fig. 1b, $p \leq 0.001{ }^{\star}$ and $p \leq 0.0001$ ) ).

\section{$\mathrm{BM} \mathrm{CD4}^{+} \mathbf{T}$ cells are elevated in female mice irrespective of mouse strains}

$\mathrm{BM} \mathrm{CD} 4^{+} \mathrm{T}$ cells were significantly elevated in females of BALB/cAnNCr (Fig. 1a, $p \leq 0.01$ ). BM CD4 $4^{+} \mathrm{T}$ cells were also elevated in females of the other two strains; however, the trend was not significant. Similarly, splenic $\mathrm{CD}^{+}$ $\mathrm{T}$ cells were also higher in $\mathrm{BALB} / \mathrm{cAnNCr}$ females, although the trend was not statistically significant. This trend was reversed for splenic $\mathrm{CD} 4^{+} \mathrm{T}$ cells for C57BL/ $6 \mathrm{NCr}$ and $129 / \mathrm{SvHsd}$, in which males had a higher percentage of $\mathrm{CD}^{+}{ }^{+} \mathrm{T}$ cells in comparison to females (Fig. 1b, n.s. for C57BL/6NCr; $p \leq 0.0001$ for $129 / \mathrm{SvHsd}$ ).

\section{$\mathrm{CD8}^{+} \mathrm{T}$ cells are elevated in $\mathrm{BM}$ of $\mathrm{C} 57 \mathrm{BL} / 6 \mathrm{NCr}$ and reduced in spleens of 129/SvHsd mice}

The BM represents a specialized niche for development of memory $\mathrm{CD}^{+} \mathrm{T}$ cells, whereas spleen is a recognized organ for being abundant in all $\mathrm{T}$ cells $[5,6]$. The percentages of $\mathrm{CD} 8^{+} \mathrm{T}$ cells in $\mathrm{BM}$ and spleen ranged from $4.28 \%$ to $5.60 \%$ and $11.51 \%$ to $21.39 \%$, respectively, in the three murine strains analyzed (Fig. 1c, d). C57BL/6NCr females exhibited a significantly higher baseline BM CD8 ${ }^{+}$ $\mathrm{T}$ cell population in comparison to $\mathrm{BALB} / \mathrm{cAnNCr}$ and $129 /$ SvHsd (Fig. 1c, $p \leq 0.0001$ and $p \leq 0.001$, respectively). 129/SvHsd exhibited a significantly reduced percentage of splenic $\mathrm{CD}^{+} \mathrm{T}$ cells in comparison to C57BL/ $6 \mathrm{NCr}$ and BALB/cAnNCr (Fig. 1d, $p \leq 0.00010^{\star}$ and $p \leq$ 0.01 i in $\mathrm{C} 57 \mathrm{BL} / 6 \mathrm{NCr}$ and $p \leq 0.0001$ for both genders in $\mathrm{BALB} / \mathrm{cAnNCr}$ ). In contrast to $\mathrm{BM}$, splenic $\mathrm{CD} 8^{+} \mathrm{T}$ cells in $\mathrm{C} 57 \mathrm{BL} / 6 \mathrm{NCr}$ females were significantly reduced in comparison to BALB/cAnNCr females (Fig. 1d, $p \leq 0.01$ ). In $\mathrm{BALB} / \mathrm{cAnNCr}$, splenic $\mathrm{CD} 8^{+} \mathrm{T}$ cells were significantly elevated in female counterparts in comparison to their male counterparts (Fig. 1d, $p \leq 0.01$ ).

\section{$\mathrm{BM} \mathrm{CD4}^{+}$regulatory $\mathrm{T}$ cells exhibit significant gender-dependent variation in all strains}

$\mathrm{CD}^{+}{ }^{+}$regulatory $\mathrm{T}$ cells have been suggested to reside in $\mathrm{BM}$ sinuses and under homeostatic conditions can be found mainly in thymus, peripheral blood, lymph nodes, and spleen $[5,43]$. In $\mathrm{BM}, \mathrm{CD}^{+}$regulatory $\mathrm{T}$ were present in the range of $0.24-0.51 \%$ across three strains (Fig. 1e, f). Like $\mathrm{CD} 4^{+} \mathrm{T}$ cells, the $\mathrm{CD} 4^{+}$Tregs were also elevated in splenic tissue in comparison to $\mathrm{BM}$ with a range of $2.04-3.15 \%$ (Fig. 1f). BM CD4 ${ }^{+}$Tregs were significantly increased in male $\mathrm{BALB} / \mathrm{cAnNCr}$ with respect to male $129 /$ SvHsd mice; however, this trend was reversed among females (Fig. 1e, $p \leq 0.0010^{\star}$ and $p \leq 0.05$ ). We also observed some similarities in strain-specific distribution within different categories of splenic $\mathrm{T}$ cells that were analyzed. All three classes of splenic $\mathrm{T}$ cells analyzed $\left(\mathrm{CD}^{+}, \mathrm{CD}^{+}\right.$, and $\mathrm{CD} 4^{+}$Tregs cells) were significantly reduced in female $\mathrm{C} 57 \mathrm{BL} / 6 \mathrm{NCr}$ and $129 / \mathrm{SvHsd}$ mice in comparison to female BALB/cAnNCr mice (Fig. 1f, $p \leq 0.0001$ for $\mathrm{C} 57 \mathrm{BL} / 6 \mathrm{NCr}$ and $p \leq 0.01$ for $129 / \mathrm{SvHsd}$ ). Splenic $\mathrm{CD}^{+}$Tregs were significantly increased in male $129 / \mathrm{SvHsd}$ in comparison to male C57BL/6NCr, a trend that was also observed in splenic $\mathrm{CD} 4^{+} \mathrm{T}$ cells (Fig. 1b, $\mathrm{f}$, $p \leq 0.0001$ and $p \leq 0.05$, respectively).

Although there were many gender-specific variations in Treg frequency within both organ sites, unlike BM CD4 $4^{+}$ $\mathrm{T}$ cells, there was no preponderance of one gender over the other (Fig. 1e, f). Whereas BM CD4 $4^{+}$Tregs were higher in females of $\mathrm{C} 57 \mathrm{BL} / 6 \mathrm{NCr}$ and $129 / \mathrm{SvHsd}$, the trend was reversed for BALB/cAnNCr (Fig. 1e, $p \leq 0.0001$ for 129/ SvHsd). In spleen these trends appeared to be reversed for each case, being significant in BALB/cAnNCr (Fig. 1f, $p \leq 0.01$ ). Overall, the differences within various subsets of $\mathrm{T}$ cells suggest that even under normal physiological conditions, $\mathrm{T}$ cells exhibit significant strain- and genderdependent variation, which could be an important factor to consider when designing in vivo experiments. For instance, gender- and strain-specific differences in $\mathrm{CD}^{+} \mathrm{T}$ cells 
A

CD4 ${ }^{+} \mathrm{T}$ cells - Bone
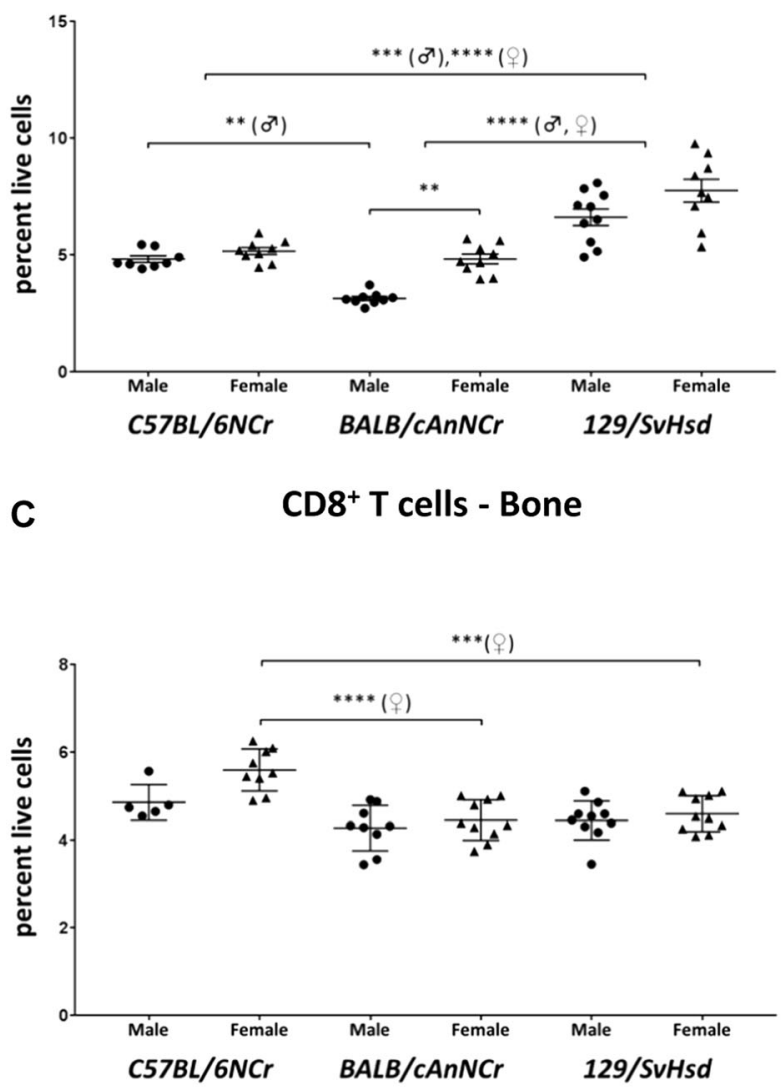

$\mathbf{E}$

CD4 ${ }^{+}$regulatory T cells - Bone

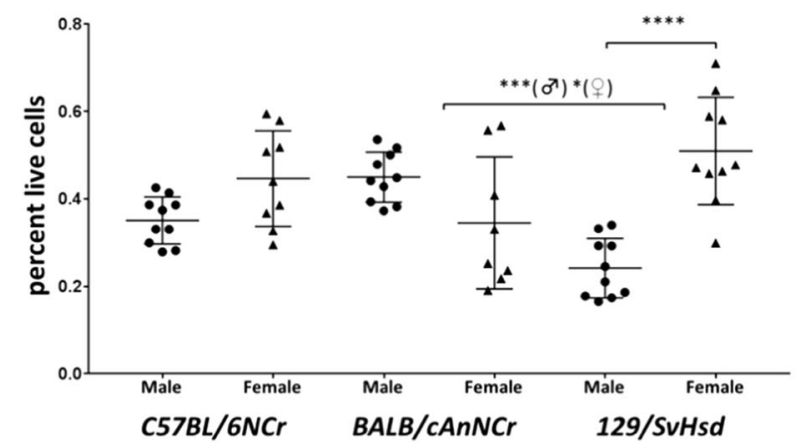

Fig. 1 Immune cell distribution of $\mathrm{T}$ cells in C57BL/6NCr, BALB/ cAnNCr, and 129/SvHsd male and female mice. Cell suspensions from $\mathrm{BM}$ and spleen were isolated from 8-week-old mice. The cells were stained with $\mathrm{CD} 3$ and $\mathrm{CD} 4$ antibodies for $\mathrm{CD} 4{ }^{+} \mathrm{T}$ cells, CD3 and CD8 antibodies for $\mathrm{CD}^{+} \mathrm{T}$ cells, and CD3, CD4, CD25, and FoxP3 antibodies for Tregs. The cells were then subjected to flow cytometry to determine cell-type percentages. a $\mathrm{CD} 4^{+} \mathrm{T}$ cells in $\mathrm{BM}(n \geq 8)$.
B

$\mathrm{CD}^{+} \mathrm{T}$ cells - Spleen

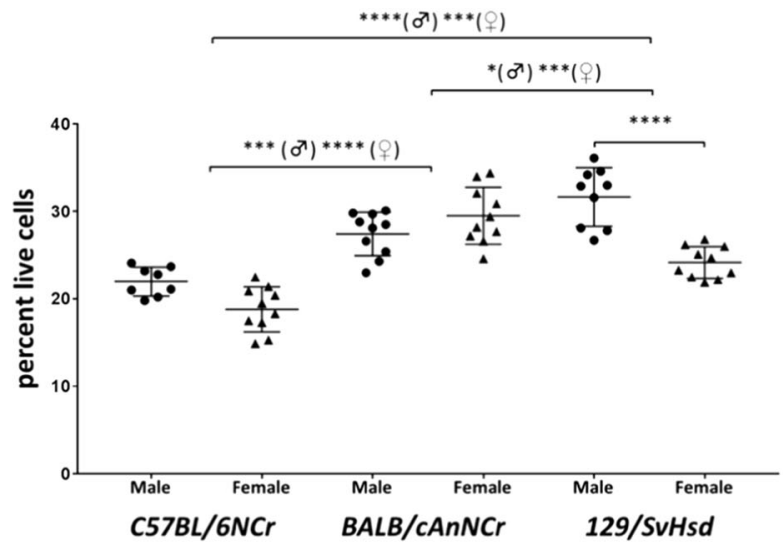

D

CD8 ${ }^{+}$T cells - Spleen

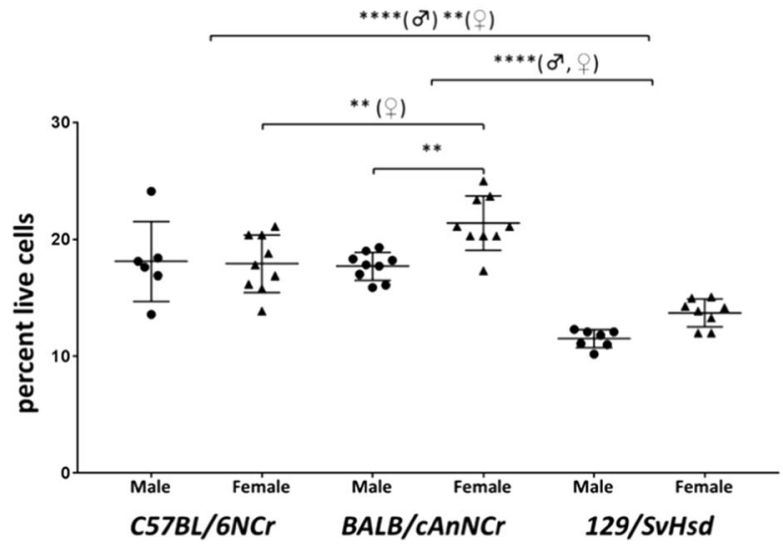

$\mathbf{F}$

$\mathrm{CD4}^{+}$regulatory $\mathrm{T}$ cells - Spleen

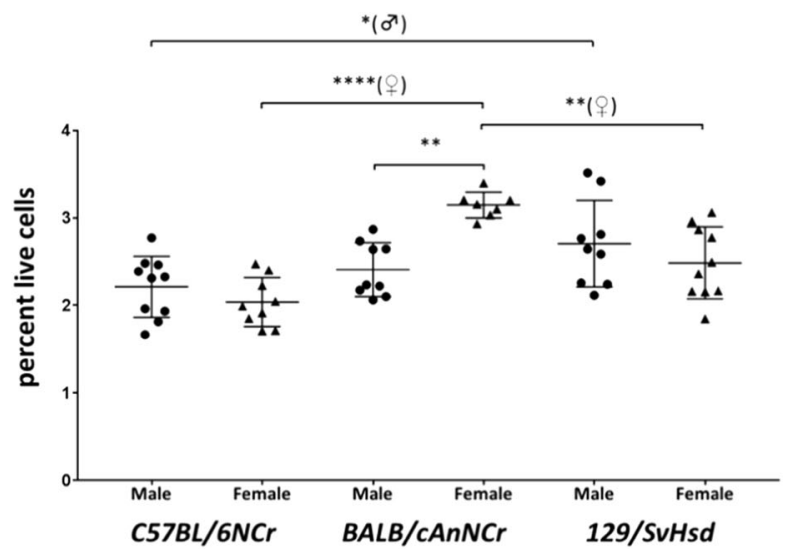

b $\mathrm{CD}^{+} \mathrm{T}$ cells in the spleen $(n \geq 8)$. $\mathbf{c ~ C D} 8^{+} \mathrm{T}$ cells in $\mathrm{BM}(n \geq 5)$. d $\mathrm{CD}^{+} \mathrm{T}$ in spleen $(n \geq 6)$. e CD4 ${ }^{+}$Tregs in $\mathrm{BM}(n \geq 8)$. f CD4 ${ }^{+}$ Tregs in spleen $(n \geq 7)$. Results, shown as scatter plots, depict average cell percentages (percent of live cells). Error bars denote SEM. Each dot represents the value from a single mouse. (o) and (o) represent male and female mice, respectively. $* p \leq 0.05, * * p \leq 0.01$, $* * * p \leq 0.001, * * * * p \leq 0.0001$ 
A

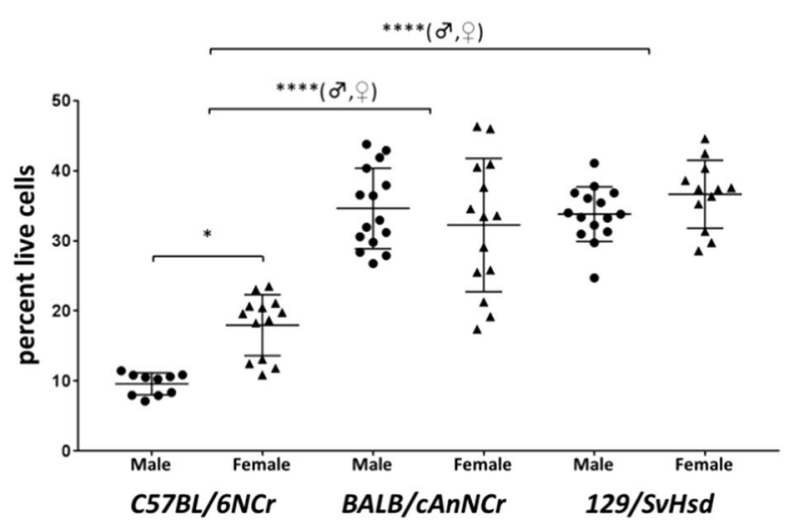

C

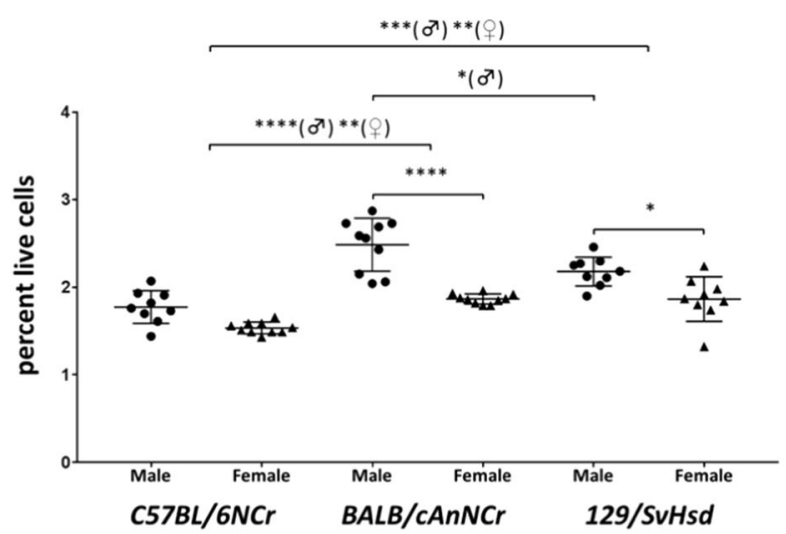

Fig. 2 Immune cell distribution of B and NK cells in C57BL/6NCr, $\mathrm{BALB} / \mathrm{cAnNCr}$, and 129/SvHsd for male and female mice. Cell suspensions from $\mathrm{BM}$ and spleen were isolated from 8-week-old mice. The cells were stained with B220 and CD19 antibodies for B cells, and CD3 and NKp46 antibodies for NK cells. The cells were then subjected to flow cytometry to determine cell-type percentages. a B cells

might have important consequences on CTL response to either pathogenic or oncogenic stimuli. Similarly, differences in $\mathrm{CD}^{+}{ }^{+}$Tregs could impact studies where immunosuppression plays a significant role.

\section{B cells are significantly reduced in C57BL/6NCr mice}

B cells can function as plasma cells that secrete antibodies against different antigens; or as memory B cells, which retain immunological memory of the antigen in order to proliferate and differentiate into plasma cells under appropriate stimuli [42]. Within all the cell types analyzed, B cells were the most abundant immune cell type in both BM and spleen (Fig. 2a, b). Among the mouse strains characterized, $B$ cell percentages in $\mathrm{BM}$ and spleen were between $9.59 \%$

\section{B B cells - Spleen}

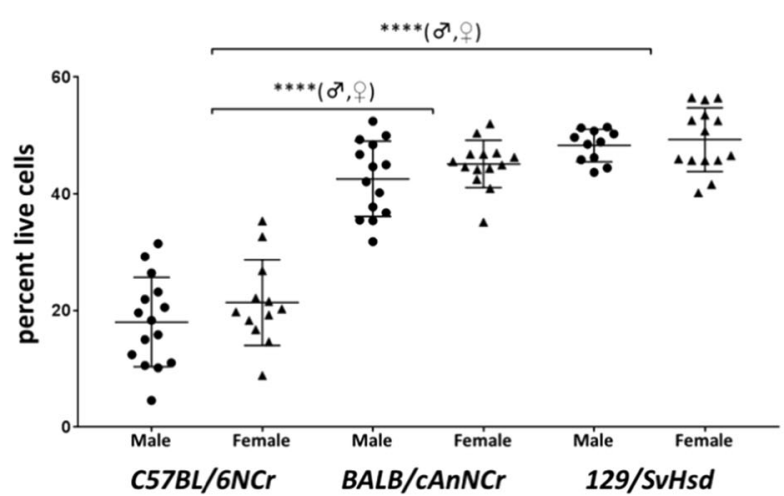

D NK cells - Spleen

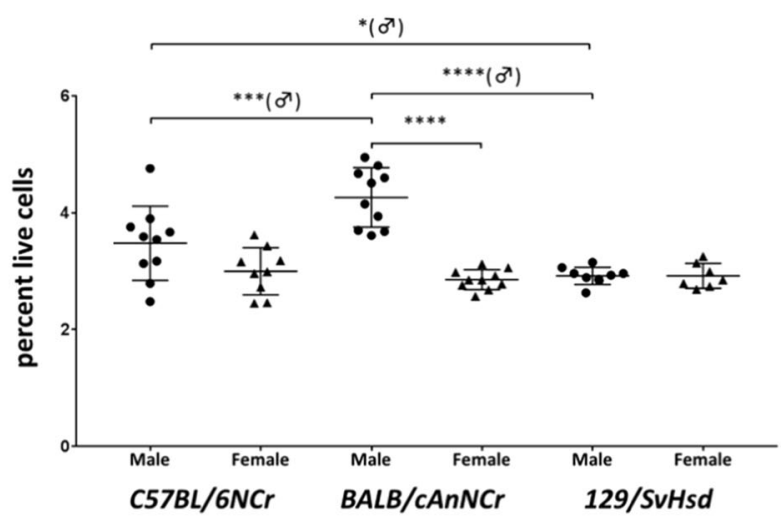

in BM $(n \geq 10)$. b B cells in the spleen $(n \geq 11)$. $\mathbf{c ~ N K}$ cells in BM $(n \geq$ 9). d NK cells in the spleen $(n \geq 7)$. Results are shown as scatter plots depicting average cell percentages (percent of live cells). Error bars denote SEM. Each dot represents the value from a single mouse. (o) and (ㅇ) represent male and female mice, respectively. $* * p \leq 0.01$, $* * * p \leq 0.001, * * * * p \leq 0.0001$

and $36.65 \%$ and $18.01 \%$ and $49.30 \%$, respectively (Fig. 2a, b). In both organs sites, we observed a significantly reduced $\mathrm{B}$ cell percentage in $\mathrm{C} 57 \mathrm{BL} / 6 \mathrm{NCr}$ in comparison to the other two strains (Fig. 2a, b, $p \leq$ 0.0001). In $\mathrm{BM}, \mathrm{C} 57 \mathrm{BL} / 6 \mathrm{NCr}$ cells also exhibited significant gender-specific divergence, as female mice exhibited almost twice the amount of B cells in comparison to males (Fig. 2a, $p \leq 0.05$ ).

\section{Natural killer cells are significantly elevated in male BALB/cAnNCr mice}

Natural killer (NK) cells play a critical role in anti-tumor surveillance, elimination of virally infected cells, and graftvs.-host disease (GVHD) [44]. NK cell percentages in BM and spleen were between $1.54 \%$ and $2.49 \%$ and $2.86 \%$ and 
$4.26 \%$, respectively (Fig. 2c, d). In the BM, NK cells were significantly reduced in C57BL/6NCr (Fig. 2c, $p \leq 0.0001$ क, $p \leq 0.01$ o for BALB/cAnNCr and $p \leq 0.001 \sigma^{\star}, p \leq 0.01$ 우 for 129/SvHsd). BALB/cAnNCr males had higher NK numbers than 129/SvHsd in the BM (Fig. 2c, $p \leq 0.05$ ). In the spleen, male BALB/cAnNCr mice had higher NK numbers than either C57BL/6NCr or 129/SvHsd (Fig. 2d, $p \leq 0.001$ and $p \leq 0.0001$, respectively). Additionally, male C57BL/6NCr had higher splenic NK cells than male 129/SvHsd (Fig. 2d, $p \leq 0.05$ ). Examining differences by gender, male BALB/ cAnNCr and 129/SvHsd had higher NK cell numbers in the $\mathrm{BM}$ (Fig. 2c, $p \leq 0.0001$ and $p \leq 0.05$, respectively), while only male BALB/cAnNCr had significantly higher NK cell numbers compared to female counterparts in the spleen (Fig. $2 \mathrm{~d}, p \leq 0.0001$ ).

\section{iMCs exhibit a male bias in BM and spleen}

iMCs exist in the BM as precursors for dendritic cells, macrophages, and neutrophils [45]. In the three strains that were assessed, iMC percentages in BM and spleen ranged from $13.46 \%$ to $28.17 \%$ and $1.12 \%$ to $2.33 \%$, respectively (Fig. 3). BM iMCs were significantly elevated in C57BL/6NCr (Fig. 3a, $p \leq 0.00010^{\star}, p \leq 0.01$ 웅 for both BALB/cAnNCr and 129/SvHsd). BALB/cAnNCr female mice exhibited significantly reduced splenic iMCs in comparison to female C57BL/6NCr and 129/ SvHsd mice (Fig. 3b, $p \leq 0.0001$ ). In the $\mathrm{BM}$, male C57BL/6NCr mice had significantly higher iMCs than female counterparts (Fig. 3a, $p \leq 0.0001$ ). In the spleen, male $\mathrm{BALB} / \mathrm{cAnNCr}$ mice exhibited greater iMC numbers in comparison to corresponding females (Fig. 3b, $p \leq 0.0001$ ).

\section{Splenic and BM macrophages in C57BL/6NCr females are significantly elevated}

Macrophages are phagocytic immune cells residing in different tissues that function as antigen-presenting cells (APCs) [42]. Macrophages arise from monocytic lineage and differentiate in a tissue-specific manner [42]. In our studies, macrophage percentages in BM and spleen were between $3.48 \%$ and $9.30 \%$ and $0.50 \%$ and $4.58 \%$, respectively, in the three murine strains analyzed (Fig. 3c, d). We observed many interesting strain- and gender-dependent variations in macrophage population. Within the BM, C57BL/6NCr females had higher macrophage numbers than both BALB/cAnNCr and 129/ SvHsd females (Fig. 3c, $p \leq 0.0001$ and $p \leq 0.001$, respectively). BALB/cAnNCr females exhibited significantly reduced BM macrophages compared to 129/ SvHsd females (Fig. 3c, $p \leq 0.05$ ). Splenic macrophages were significantly elevated in $\mathrm{C} 57 \mathrm{BL} / 6 \mathrm{NCr}$ among both genders in comparison to BALB/cAnNCr and 129/ SvHsd (Fig. 3d, $p \leq 0.0001$ ). BM macrophages were significantly higher in male BALB/cAnNCr mice with respect to their female counterparts (Fig. $3 c, p \leq 0.0001$ ), while the only gender bias noted in the spleen was among C57BL/6NCr males which exhibited higher splenic-macrophage numbers in comparison to females of the same strain (Fig. 3d, $p \leq 0.01$ ).

\section{Myeloid dendritic cells are significantly elevated in BM of 129/SvHsd and spleen of C57BL/6NCr mice}

Dendritic cells are professional APCs that play key roles in both the innate and adaptive immunity [42, 46]. Myeloid dendritic cells $(\mathrm{mDCs})$ are specialized subsets of dendritic cells that capture, process, and present antigens on their surface to T cells $[42,46]$. Percentages of mDCs in BM and spleen ranged from $2.78 \%$ to $7.45 \%$ and $1.19 \%$ to $3.83 \%$, respectively, in the three murine strains analyzed (Fig. 3e, f). $129 / \mathrm{SvH}$ sd mice exhibited significantly higher mDCs within BM (Fig. 3e, $p \leq 0.0001$; both strains and in both genders). In contrast, splenic $\mathrm{mDCs}$ were significantly higher among C57BL/6NCr in comparison to $129 / \mathrm{SvHsd}$ (Fig. 3f, $p \leq 0.0001{ }^{\star}, p \leq 0.001$ ) $)$. Male C57BL/6NCr also exhibited significantly higher splenic mDCs in comparison to $\mathrm{BALB} / \mathrm{cAnNCr}$ mice, but in females this effect was not statistically significant among these two strains (Fig. 3f, $p \leq 0.0001)$. Splenic $\mathrm{mDC}$ were significantly elevated in male $\mathrm{BALB} / \mathrm{cAnNCr}$ mice in comparison to male 129/SvHsd mice (Fig. 3f, $p \leq 0.05$ ). The only genderspecific variation noted was a higher splenic mDC population within C57BL/6NCr males (Fig. 3f, $p \leq 0.0001$ ).

\section{Plasmacytoid dendritic cells exhibit a strong gender difference in BM of all three strains and in C57BL/ $6 \mathrm{NCr}$ splenocytes}

Plasmacytoid dendritic cells (pDCs) are specialized dendritic cells that perform a critical role in both innate and adaptive immunity [42, 47]. Found in circulating blood and peripheral lymphoid organs, pDCs secrete IFNs, and mediate anti-viral immunity and anti-inflammatory responses $[42,47]$. In our studies, pDC percentages in BM and spleen ranged from $1.06 \%$ to $1.93 \%$ and 0.12 to $0.55 \%$, respectively, in the three murine strains analyzed (Fig. 4a, b). Within the BM, C57BL/6NCr male mice exhibited a significantly higher pDC population in comparison to BALB/ cAnNCr male mice (Fig. $4 \mathrm{a}, p \leq 0.01$ ). pDCs were significantly elevated in $\mathrm{C} 57 \mathrm{BL} / 6 \mathrm{NCr}$ female mice in comparison to $129 / \mathrm{SvHsd}$ female mice (Fig. $4 \mathrm{a}, p \leq 0.01$ ). pDCs were also significantly elevated in $\mathrm{C} 57 \mathrm{BL} / 6 \mathrm{NCr}$ male mice in comparison to BALB/cAnNCr male mice (Fig. 4a, $p \leq 0.01)$. On the other hand, C57BL/6NCr mice had a 
A

iMCs - Bone

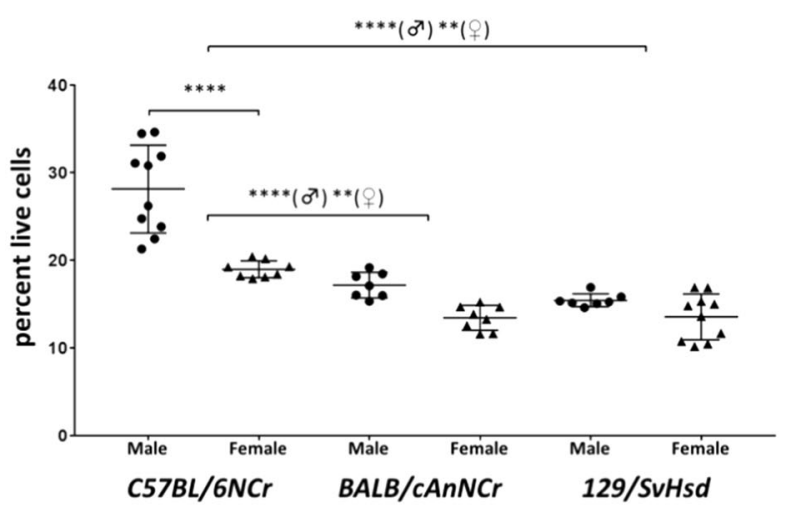

C Macrophages - Bone

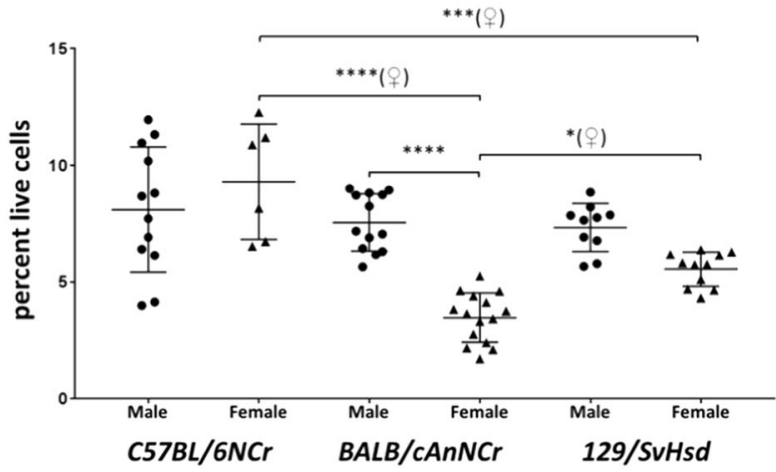

E

mDCs - Bone

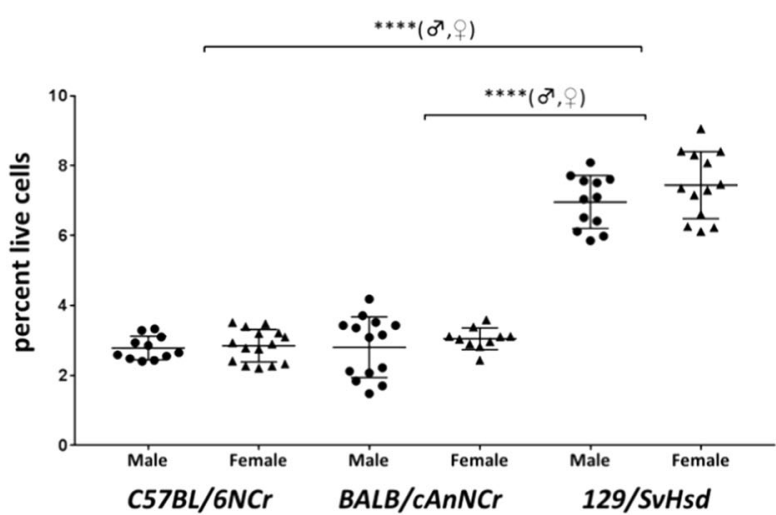

Fig. 3 Immune cell distribution of immature myeloid cells (iMCs), macrophages, and myeloid dendritic cells (mDCs) in C57BL/6NCr, BALB/cAnNCr, and 129/SvHsd for male and female mice. Cell suspensions from $\mathrm{BM}$ and spleen were isolated from 8-week-old mice. The cells were stained with CD11b, F4/80, and CD68 antibodies for macrophages, CD11b and Gr-1 antibodies for iMCs, and CD11b and CD11c antibodies for mDCs. Ly6B was used as negative marker for iMCs. The cells were then subjected to flow cytometry to determine
B

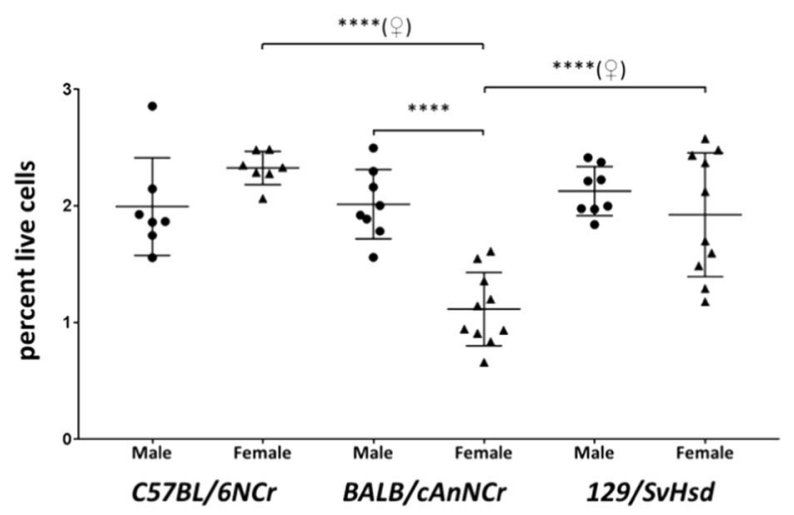

D Macrophages - Spleen
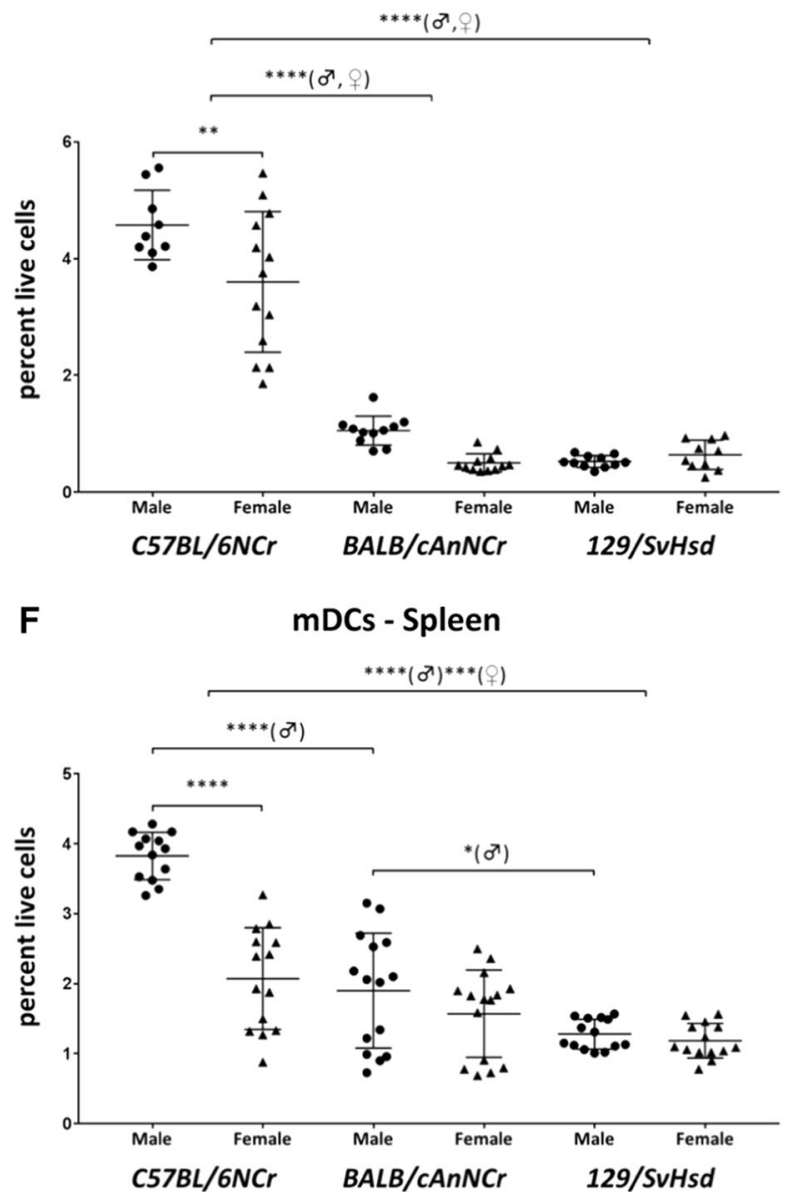

cell-type percentages. a iMCs in BM $(n \geq 7)$. b iMCs in the spleen $(n \geq$ 7). c Macrophages in BM $(n \geq 6)$. d Macrophages in spleen $(n \geq 9)$. $\mathbf{e}$ mDCs in BM $(n \geq 10)$. f mDCs in the spleen $(n \geq 13)$. Results are shown as scatter plots depicting average cell percentages (percent of live cells). Error bars denote SEM. Each dot represents the value from a single mouse. ( $\left.\sigma^{\star}\right)$ and (o) represent male and female mice, respectively. $* * p \leq 0.01, * * * p \leq 0.001, * * * * p \leq 0.0001$ 
A

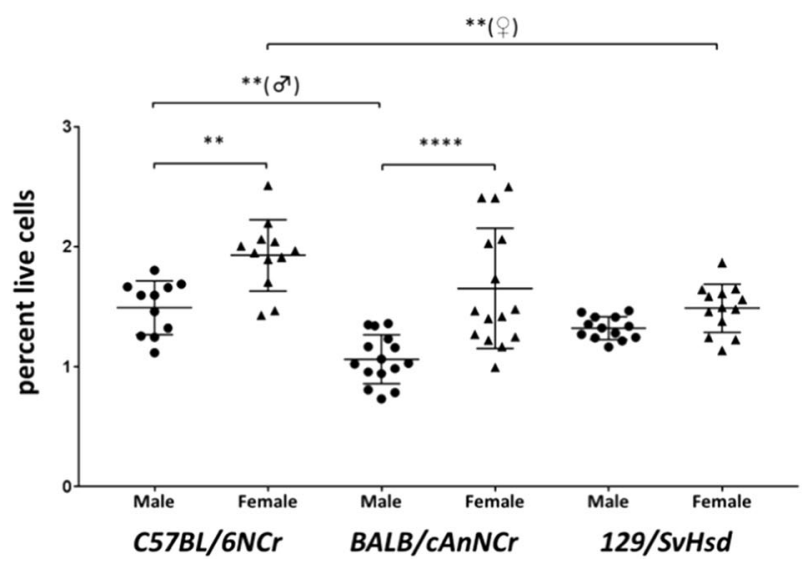

Fig. 4 Immune cell distribution of plasmacytoid dendritic cells in C57BL/6NCr, BALB/cAnNCr, and 129/SvHsd male and female mice. Cell suspensions from BM and spleen were isolated from 8-week-old mice. The cells were stained with CD11c, B220, and Siglec H antibodies. CD11b antibody was included as a negative marker for pDCs. The cells were then subjected to flow cytometry to determine cell-type
B

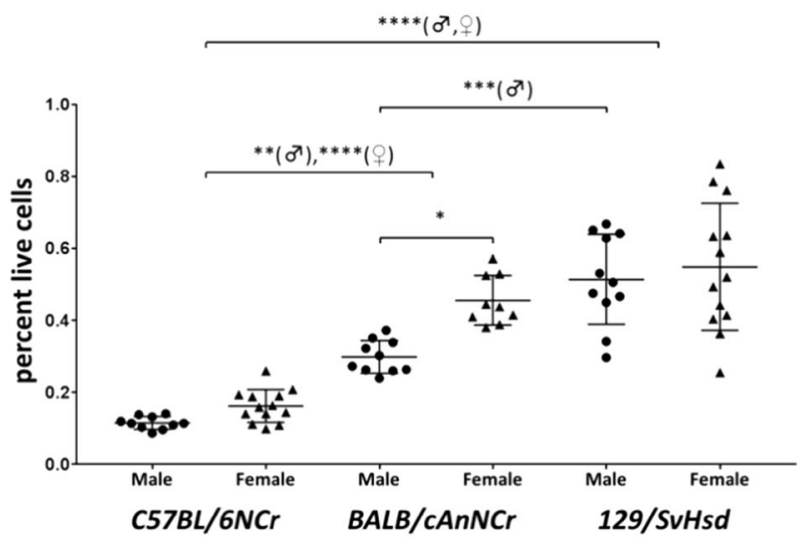

percentages. a pDCs in BM $(n \geq 11)$. b pDCs in the spleen $(n \geq 9)$. Results are shown as scatter plots depicting average cell percentages (percent of live cells). Error bars denote SEM. Each dot represents the value from a single mouse. $\left({ }^{\star}\right)$ and (o) represent male and female mice respectively. ${ }^{*} p \leq 0.05, * * p \leq 0.01, * * * p \leq 0.001, * * * * p \leq 0.0001$

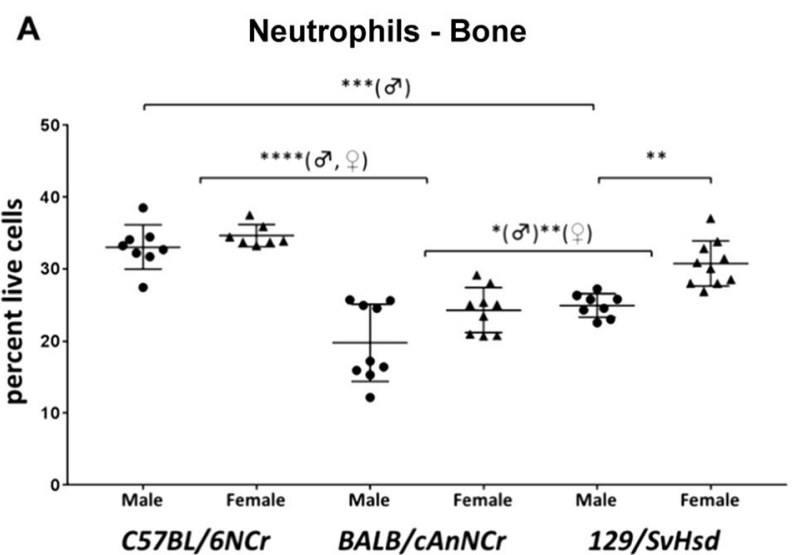

Fig. 5 Immune cell distribution of neutrophils in C57BL/6NCr, BALB/cAnNCr, and 129/SvHsd for male and female mice. Cell suspensions from $\mathrm{BM}$ and spleen were isolated from 8-week-old mice. The cells were stained with CD11b, Gr-1, and Ly6b antibody. F4/80 antibody was included as a negative marker for neutrophils. The cells were then subjected to flow cytometry to determine cell-type

significantly reduced baseline percentage of pDCs within the spleen (Fig. 4b, $p \leq 0.010, p \leq 0.0001$ 우 for BALB/ cAnNCr and $p \leq 0.0001$ in both genders for $129 / \mathrm{SvHsd}$ ). Splenic pDCs were significantly elevated in $129 / \mathrm{SvHsd}$ female mice in comparison to BALB/cAnNCr female mice (Fig. $4 b, p \leq 0.001$ ). There was clear gender-specific difference in pDCs within the BM, with females from C57BL/ $6 \mathrm{NCr}$ and $\mathrm{BALB} / \mathrm{cAnNCr}$ strains exhibiting a significantly higher $\mathrm{pDC}$ population in comparison to the corresponding males (Fig. $4 \mathrm{a}, p \leq 0.01$ and $p \leq 0.0001$, respectively). This
B Neutrophils - Spleen

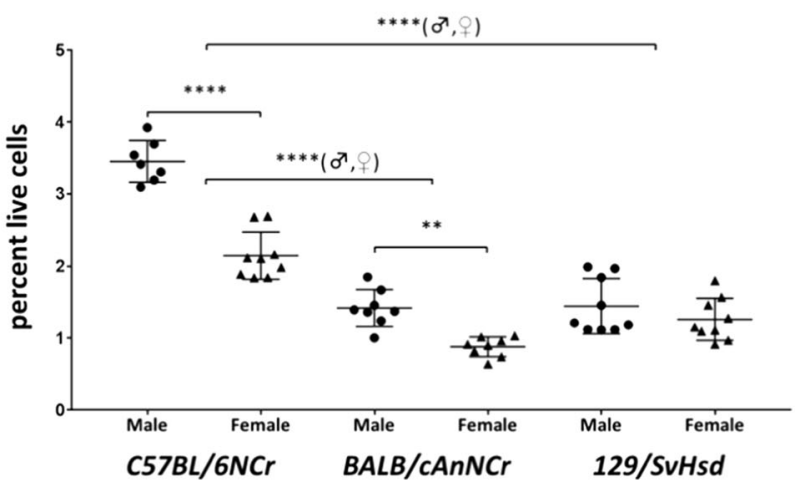

percentages. a Neutrophils in $\mathrm{BM}(n \geq 7)$. b Neutrophils in spleen $(n \geq$ 7). Results are shown as scatter plots depicting average cell percentages (percent of live cells). Error bars denote SEM. Each dot represents the value from a single mouse. ( $\left.\sigma^{*}\right)$ and (o) represent male and female mice respectively. $* p \leq 0.05, * * p \leq 0.01$, ***p $\leq 0.001$, **** $p \leq$ 0.0001

trend was observed to be significant in the spleen for BALB/cAnNCr, with females being higher than male counterparts (Fig. $4 b, p \leq 0.05$ ).

\section{Neutrophils are elevated in $\mathrm{C57BL} / 6 \mathrm{NCr}$ and exhibit gender-specific variation in both BM and spleen}

Neutrophils are the most abundant granulocytes, comprising $40-75 \%$ of peripheral blood immune cells [42, 48]. Formed within the $\mathrm{BM}$, neutrophils play critical roles in 
phagocytosis of invading microbes [42, 48]. Neutrophil percentages were between $19.81 \%$ and $34.67 \%$ and $0.88 \%$ and $3.45 \%$ in $\mathrm{BM}$ and spleen, respectively in the three murine strains analyzed (Fig. 5a, b). In BM, C57BL/6NCr male mice exhibited a significantly higher neutrophil population in comparison to $129 / \mathrm{SvHsd}$ (Fig. $5 \mathrm{a}, p \leq 0.001$ ). This trend was observed in both genders when comparing C57BL/6NCr and BALB/cAnNCr (Fig. 5a, $p \leq 0.0001$ ). Similarly splenic neutrophils of $\mathrm{C} 57 \mathrm{BL} / 6 \mathrm{NCr}$ were significantly higher than both BALB/cAnNCr and 129/SvHsd (Fig. 5b, $p \leq 0.0001$ ). 129/SvHsd exhibited a significantly higher BM neutrophil percentage in comparison to BALB/ cAnNCr strain (Fig. 5a, $p \leq 0.05 \delta^{\star}, p \leq 0.01$ \%). Within the BM, gender bias was observed among $129 / \mathrm{SvHsd}$, with females higher than males (Fig. 5a, $p \leq 0.01$ ). Within the spleen, female C57BL/6NCr and BALB/cAnNCr mice had higher neutrophils in comparison to males (Fig. 5b, $p \leq$ 0.0001 and $p \leq 0.01$, respectively).

\section{Discussion}

The methodology of conducting immunophenotypic analysis of different cell populations can vary considerably from one study to another based on the endpoints and the goals of the study [24, 29, 31, 32]. For instance, different studies may report immune cell percentages in distinct ways based on normalization strategies and gating methods (e.g. percent of total lymphocytes, percent of total $\mathrm{T}$ cells, percent of total $\mathrm{CD}^{+} \mathrm{T}$ cells, absolute $\mathrm{T}$ cell number, etc.), which can result in substantial differences in the interpretation of the results even while evaluating the same cell type [31, 32]. Importantly, although some studies have determined immune cell frequencies in peripheral blood, only minimal inference is gleaned for the expected immune profile in BM and spleen from such studies [12-15]. BM and spleen serve as major reservoirs of immune cells in mice and maintain immune homeostasis in peripheral tissue [49]. As observed in this detailed study, a summary of immune cell distribution in $\mathrm{BM}$ and spleen of C57BL/ 6NCr, BALB/cAnNCr, and 129/SvHsd mice indicates that this homeostasis varies significantly between the commonly used in vivo mouse models (Fig. 6; [50, 51]). In the absence of an organ-specific baseline immune profile in normal mice, a comparative analysis within different strains and/or gender following experimental stimuli can be challenging, and may impose inherent bias while extrapolating experimental data. Therefore, there is a pressing need for unified studies cataloging baseline immune cell distribution in different strains of mice [31].

From the results of the present study, it is encouraging to find that overall immune cell percentages observed by us in $\mathrm{BM}$ and spleen was in close agreement to previously published murine and human studies examining other organs [12-15, 25-27, 45, 52-55]. Our comprehensive analysis reveals many interesting findings. For instance, total neutrophils and splenic macrophages are significantly higher in C57BL/6NCr, whereas total B cells are lower, compared to BALB/cAnNCr and $129 / \mathrm{SvHsd}$ mice. Similarly, the significantly higher number of $\mathrm{mDCs}$ in $\mathrm{BM}$ from $129 / \mathrm{SvHsd}$ is also apparent. There are many critical trends that become apparent upon careful examination of immune cell subtypes through the present study. The identified strain-specific variation, both in $\mathrm{CD} 4^{+}$and $\mathrm{CD} 8^{+} \mathrm{T}$ cells, underscores the importance of this difference when using different strains and gender of mice in preclinical studies mimicking human diseases, including cancer, which responds differently based on the profile of the adaptive immune system [50, 51]. Notably, C57BL/6NCr and $\mathrm{BALB} / \mathrm{cAnNCr}$ mice, which exhibited strain- and genderspecific differences in $\mathrm{T}$ cell profile, are recognized as prototypical Th1- and Th2 responders, respectively [56]. These strains are known to have distinct differences in sensitivity to pathophysiological challenge and kinetics of tumor growth [56-61]. Given the observed differences in T cell profile observed by us across these strains, it can be surmised that mice strains with differing baseline $\mathrm{T}$ cell profiles would respond differently in preclinical evaluation of new immunotherapeutic drugs [50,51, 57,60].

Many in vivo murine studies are often conducted in female mice due to ease of handling [62]. Due to inherent genetic characteristics favoring tumor susceptibility, BALB/ cAnNCr mice are frequently employed in cancer-related studies [36, 37]. We found that within BALB/cAnNCr, female mice had significantly elevated $\mathrm{CD}^{+} \mathrm{T}$ cells. This higher baseline of $\mathrm{CD}^{+} \mathrm{T}$ cells noted in female mice could skew observations related to tumor kinetics, leading to contrasting experimental results dependent on gender. We also observed considerable gender-specific differences in $\mathrm{BM} \mathrm{CD} 4^{+}$Tregs. Th2 cytokines in bronchoalveolar lavage fluid are significantly elevated in females of certain mouse strains upon ovalbumin challenge [63]. $\mathrm{CD}^{+}$Tregs, which are recognized as suppressors of inflammation and critical mediators in airway hyperresponsiveness, exhibited significant gender-dependent difference in this study, suggesting that baseline, gendervariation in Tregs may skew the results in murine asthma studies [64]. Overall, we observed both strain- and genderspecific differences in baseline $\mathrm{T}$ cell population, both of which can skew experimental results of in vivo pathophysiological studies and lead to discordance within clinical results [61].

We identified that C57BL/6NCr exhibited a dramatically reduced $\mathrm{B}$ cell percentage with respect to the other two strains. Interestingly, our results demonstrate B cells to be elevated in $\mathrm{BALB} / \mathrm{cAnNCr}$ (Th2 responder) compared to 
A

\section{C57BL/6NCr male}

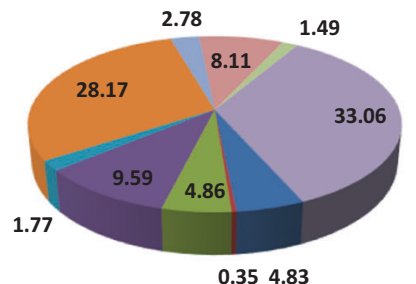

C57BL/6NCr female

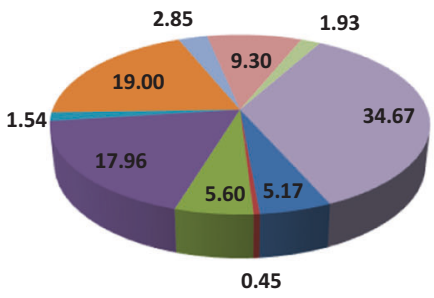

B

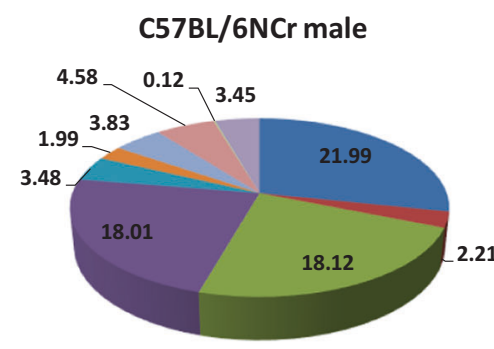

C57BL/6NCr female

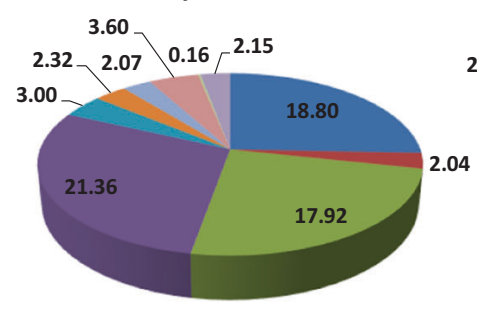

Bone

\section{Balb/cAnNCr male}

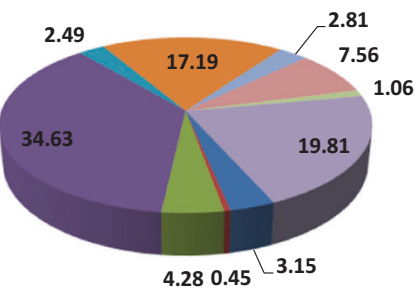

Balb/cAnCr female

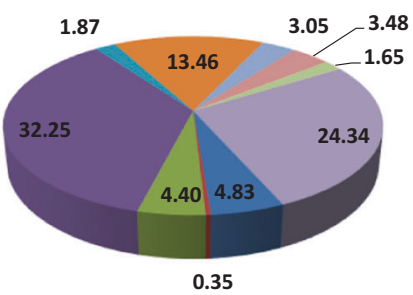

Spleen

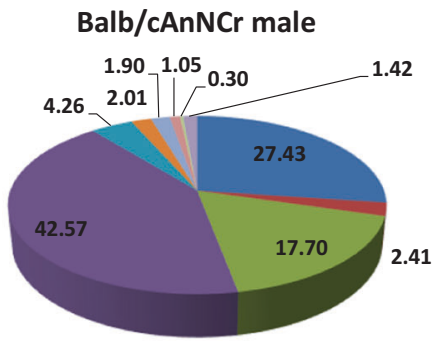

Balb/cAnNCr female

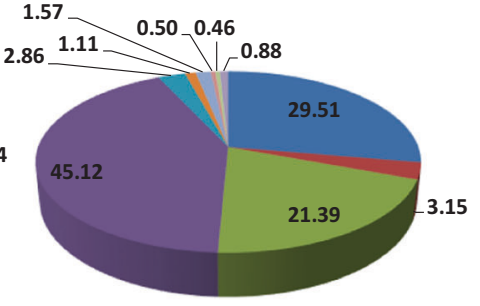

$129 /$ SvHsd male

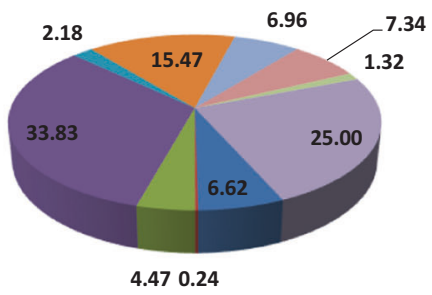

$129 /$ SvHsd female

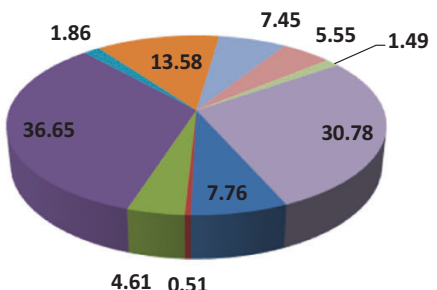

4.610 .51

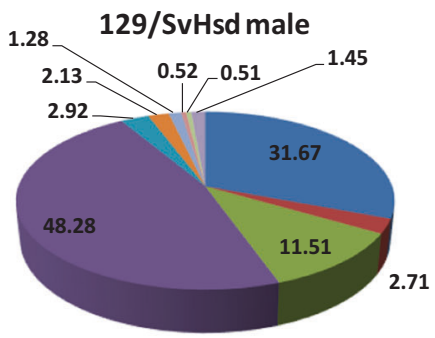

$129 /$ SvHsd female

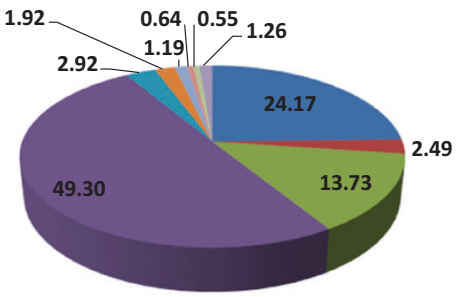

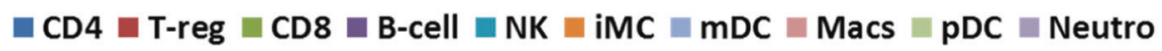

Fig. 6 Strain- and gender-specific immune cell distribution of critical cell types in three mouse strains commonly used in preclinical research. a Pie charts representing the frequency of $\mathrm{CD} 4^{+}$and $\mathrm{CD} 8^{+}$ $\mathrm{T}$ cells, Tregs, B cells, NK cells, iMCs, pDCs, mDCs, macrophages, and neutrophils in BM cells for C57BL/6NCr, BALB/cAnNCr, and $129 / \mathrm{SvHsd}$ mice. The upper three panels represent distribution in male

C57BL/6NCr (Th1 responder). In this regard, it is noteworthy that a study documented that Th2 responders preferentially activate B cells [65]. Studies have demonstrated mice and lower panels depict similar distribution in female mice. b Strain- and gender-specific immune cell distribution for $\mathrm{CD}^{+}$and CD8 ${ }^{+} \mathrm{T}$ cells, Tregs, B cells, NK cells, iMCs, pDCs, mDCs, macrophages, and neutrophils in spleens of C57BL/6NCr, BALB/cAnNCr, and $129 / \mathrm{SvHsd}$ mice. The upper and lower three panels represent distribution in male and female mice, respectively

that $\mathrm{BALB} / \mathrm{cAnNCr}$ mice exhibit a higher IgG titer than C57BL/6NCr following infection with T. cruzi [66]. Strainspecific benchmarking of $\mathrm{B}$ cell percentage in healthy 
organisms could prove useful for selecting appropriate murine models to study lymphomas and autoimmune diseases where the B cell population is significantly altered [67]. We also noticed both inter-strain and intra-strain gender differences in NK cells. In light of these observations, it is notable that different strains of inbred mice, including $\mathrm{C} 57 \mathrm{BL} / 6 \mathrm{NCr}$ and $\mathrm{BALB} / \mathrm{cAnNCr}$, exhibit marked differences in susceptibility to MCMV infection based on their NK cell profile $[68,69]$.

We observed distinct gender bias in BALB/cAnNCr, with males displaying a higher percentage of iMCs both in $\mathrm{BM}$ and spleen. In fact, within the BM, the males were found to have higher iMCs in comparison to their female counterparts. Under the influence of tumor-derived trophic factors such as transforming growth factor- $\beta$, interleukin- $1 \beta$ (IL-1 $\beta$ ), IL-6, IL-10, and VEGF, CD11b $\mathrm{br}^{+}$cells lose their capacity to differentiate and inhibit the function of $\mathrm{T}$ cells, dendritic cells, macrophages, and NK cells, thereby creating an immunosuppressive pro-tumorigenic environment [45]. It can be inferred that a baseline difference in iMCs impacts general immune-surveillance and is a critical factor mediating differences in tumor kinetics among mouse strains [70]. It was recently shown that iMCs directly contribute to skin tumor development by recruiting IL-17-producing $\mathrm{CD} 4^{+} \mathrm{T}$ cells [71]. Differences in baseline iMC population could in principle also impact MDSC profile upon pathological challenge and result in varying immunosuppressive characteristics based on gender and strain of the mice [70-72]. In fact, genetic background of $\mathrm{C} 57 \mathrm{BL} / 6 \mathrm{NCr}$ and $\mathrm{BALB} / \mathrm{cAnNCr}$ defines the levels of MDSCs and subsequent immunosuppressive effect following parasitic infections [72, 73]. Similarly, our findings showing gender bias within the same mouse strain suggest the need to consider baseline iMCs prior to experiments that may be affected by immunosuppression.

There were also significant strain-specific differences in splenic macrophages, with $\mathrm{C} 57 \mathrm{BL} / 6 \mathrm{NCr}$ exhibiting a higher frequency when compared to the other strains. Murine macrophage polarization and activation in response to pathogen challenge varies dramatically based on strain [74-76]. Similarly, the strain and gender differences within mDCs observed by us could impact susceptibility from infectious agents such as Brucella and Paracoccidioides [77, 78]. Antigen presentation by $\mathrm{pDC}$ is known to induce a context-dependent regulation of $\mathrm{CD}^{+} \mathrm{T}$ cell activation and $\mathrm{B}$ cell growth and differentiation $[79,80]$. Interestingly, we observed that splenic $\mathrm{CD}^{+} \mathrm{T}$ cells, pDCs, and $\mathrm{B}$ cell populations were significantly reduced in $\mathrm{C} 57 \mathrm{BL} / 6 \mathrm{NCr}$ mice. Furthermore, $\mathrm{BM} \mathrm{CD} 4^{+} \mathrm{T}$ cells, $\mathrm{CD} 8^{+} \mathrm{T}$ cells, and $\mathrm{pDC}$ sere elevated in females of all strains.

C57BL/6NCr mice exhibited significantly higher neutrophils with respect to the other two strains. Studies indicate sensitivity of $\mathrm{C} 57 \mathrm{BL} / 6 \mathrm{NCr}$ and $\mathrm{BALB} / \mathrm{cAnNCr}$ strains to infections correlate with neutrophil activity [81, 82]. Given that these mice strains are frequently employed to assess changes in infectious burden upon experimental stimuli, a baseline evaluation of neutrophil content in these strains is critical [9]. Similarly, in light of many studies that have reported strain and gender discrepancies in clinical asthma studies, considering the gender in such murine studies is also critical in evaluating neutrophil recruitment $[58,63,83]$. Results of this study indicate that it is important to evaluate a baseline immune profile of mice before embarking upon on in vivo studies as frequency of immune cell types may skew results. A rational choice of mouse strain and gender, based on immune-specificity profile, should provide better experimental readouts. While further information can be gleaned from other strains, organ sites (lymph nodes, pulmonary sites, etc.), cytokine profiles, and immune cell subtypes (Th1 vs. Th2, M1 vs. M2, etc.), this study provides an important point-of-comparison resource for studies utilizing these mice strains by highlighting critical strain- and gender-specific trends that could influence experimental results.

Acknowledgements Financial support from the National Institute of Health research grants CA184770 and AR060948, and the Department of Defense grant PR141945, is greatly acknowledged. We sincerely thank ENVIGO for providing mice for the study.

\section{Compliance with ethical standards}

Conflict of interest The authors declare that they have no conflict of interest.

\section{References}

1. Doulatov S, Notta F, Laurenti E, Dick JE. Hematopoiesis: a human perspective. Cell Stem Cell. 2012;10:120-36.

2. Dzierzak E, Speck NA. Of lineage and legacy: the development of mammalian hematopoietic stem cells. Nat Immunol. 2008;9: 129-36.

3. Plas DR, Rathmell JC, Thompson CB. Homeostatic control of lymphocyte survival: potential origins and implications. Nat Immunol. 2002;3:515-21.

4. Rathmell JC, Thompson CB. Pathways of apoptosis in lymphocyte development, homeostasis, and disease. Cell. 2002;109: Suppl:S97-107.

5. Zhao E, Xu H, Wang L, et al. Bone marrow and the control of immunity. Cell Mol Immunol. 2012;9:11-19.

6. Bronte V, Pittet MJ. The spleen in local and systemic regulation of immunity. Immunity. 2013;39:806-18.

7. Hensel JA, Khattar V, Ashton R, et al. Location of tumor affects local and distant immune cell type and number. Immun Inflamm Dis. 2017;5:85-94.

8. Juul S, Pliskin JS, Fineberg HV. Variation and information inwhite blood cell differential counts. Med Decis Making. 1984;4:69-80.

9. Sellers RS, Clifford CB, Treuting PM, et al. Immunological variation between inbred laboratory mouse strains: points to consider 
in phenotyping genetically immunomodified mice. Vet Pathol. 2012;49:32-43.

10. Bogue MA, Grubb SC. The Mouse Phenome Project. Genetica. 2004;122:71-4.

11. Grubb SC, Churchill GA, Bogue MA. A collaborative database of inbred mouse strain characteristics. Bioinformatics. 2004;20: 2857-9.

12. Chen J, Harrison DE. Quantitative trait loci regulating relative lymphocyte proportions in mouse peripheral blood. Blood. 2002;99:561-6.

13. Chen J, Flurkey K, Harrison DE. A reduced peripheral blood $\mathrm{CD} 4(+)$ lymphocyte proportion is a consistent ageing phenotype. Mech Ageing Dev. 2002;123:145-53.

14. Peters LL, Cheever EM, Ellis HR, et al. Large-scale, highthroughput screening for coagulation and hematologic phenotypes in mice. Physiol Genomics. 2002;11:185-93.

15. Petkova SB, Yuan R, Tsaih SW, et al. Genetic influence on immune phenotype revealed strain-specific variations in peripheral blood lineages. Physiol Genomics. 2008;34:304-14.

16. van de Geijn GJ, van Rees V, van Pul-Bom N, et al. Leukoflow: multiparameter extended white blood cell differentiation for routine analysis by flow cytometry. Cytometry A. 2011;79:694-706.

17. Zitvogel L, Pitt JM, Daillere R, et al. Mouse models in oncoimmunology. Nat Rev Cancer. 2016;16:759-73.

18. Sawant A, Schafer CC, Ponnazhagan S, et al. The dual targeting of immunosuppressive cells and oxidants promotes effector and memory T-cell functions against lung cancer. Oncoimmunology. 2014;3:e27401.

19. Sawant AC, Adhikari P, Narra SR, et al. Neutrophil to lymphocyte ratio predicts short- and long-term mortality following revascularization therapy for ST elevation myocardial infarction. Cardiol J. 2014;21:500-8.

20. Schafer CC, Wang Y, Hough KP, et al. Indoleamine 2,3-dioxygenase regulates anti-tumor immunity in lung cancer by metabolic reprogramming of immune cells in the tumor microenvironment. Oncotarget. 2016;7:75407-24.

21. Larson-Casey JL, Deshane JS, Ryan AJ, et al. Macrophage Akt1 kinase-mediated mitophagy modulates apoptosis resistance and pulmonary fibrosis. Immunity. 2016;44:582-96.

22. Sawant A, Deshane J, Jules J, et al. Myeloid-derived suppressor cells function as novel osteoclast progenitors enhancing bone loss in breast cancer. Cancer Res. 2013;73:672-82.

23. Levy S, Feduska JM, Sawant A, et al. Immature myeloid cells are critical for enhancing bone fracture healing through angiogenic cascade. Bone. 2016;93:113-24.

24. Guidance Development Review C, Working Group for Clinical Studies of Cancer I, Working Group for Effector Cell T. et al. 2015 Guidance on cancer immunotherapy development in earlyphase clinical studies. Cancer Sci. 2015;106:1761-71.

25. Elderman $M$, van Beek A, Brandsma E, et al. Sex impacts Th1 cells, Tregs, and DCs in both intestinal and systemic immunity in a mouse strain and location-dependent manner. Biol Sex Differ. 2016;7:21

26. Klein SL, Flanagan KL. Sex differences in immune responses. Nat Rev Immunol. 2016;16:626-38.

27. Pinchuk LM, Filipov NM. Differential effects of age on circulating and splenic leukocyte populations in C57BL/6 and BALB/c male mice. Immun Ageing. 2008;5:1.

28. Sivan A, Corrales L, Hubert N, et al. Commensal Bifidobacterium promotes antitumor immunity and facilitates anti-PD-L1 efficacy. Science. 2015;350:1084-9.

29. Li QX, Feuer G, Ouyang X, et al. Experimental animal modeling for immuno-oncology. Pharmacol Ther. 2017;173:34-46.

30. Abdullah M, Chai PS, Chong MY, et al. Gender effect on in vitro lymphocyte subset levels of healthy individuals. Cell Immunol. 2012;272:214-9.
31. Chen Z, Huang A, Sun J, et al. Inference of immune cell composition on the expression profiles of mouse tissue. Sci Rep. 2017:7:40508

32. Hahne F, Khodabakhshi AH, Bashashati A, et al. Per-channel basis normalization methods for flow cytometry data. Cytometry A. 2010;77:121-31.

33. Finak G, Langweiler M, Jaimes $M$, et al. Standardizing flow cytometry immunophenotyping analysis from the Human ImmunoPhenotyping Consortium. Sci Rep. 2016;6:20686.

34. Bashashati A, Brinkman RR. A survey of flow cytometry data analysis methods. Adv Bioinformatics. 2009;2009:584603.

35. Ostrand-Rosenberg S. Animal models of tumor immunity, immunotherapy and cancer vaccines. Curr Opin Immunol. 2004; $16: 143-50$.

36. Clapp NK, Tyndall RL, Otten JA. Differences in tumor types and organ susceptibility in BALB-c and RF mice following dimethylnitrosamine and diethylnitrosamine. Cancer Res. 1971; 31:196-8.

37. Ullrich RL. Tumor induction in BALB/c female mice after fission neutron or gamma irradiation. Radiat Res. 1983;93:506-15.

38. Mekada K, Abe K, Murakami A, et al. Genetic differences among C57BL/6 substrains. Exp Anim. 2009;58:141-9.

39. Jiang LI, Nadeau JH. 129/Sv mice-a model system for studying germ cell biology and testicular cancer. Mamm Genome. 2001; 12:89-94.

40. Clapcote SJ, Roder JC. Deletion polymorphism of Disc1 is common to all 129 mouse substrains: implications for genetargeting studies of brain function. Genetics. 2006;173:2407-10.

41. Rodgers RJ, Augar R, Berryman N, et al. Atypical anxiolytic-like response to naloxone in benzodiazepine-resistant $129 \mathrm{~S} 2 / \mathrm{SvHsd}$ mice: role of opioid receptor subtypes. Psychopharmacology. 2006;187:345-55.

42. Owen JA, Punt J, Stranford SA et al. Kuby immunology. New York: W.H. Freeman; 2013.

43. Wei S, Kryczek I, Zou W. Regulatory T-cell compartmentalization and trafficking. Blood. 2006;108:426-31.

44. Vivier E, Tomasello E, Baratin M, et al. Functions of natural killer cells. Nat Immunol. 2008;9:503-10.

45. Gabrilovich DI, Nagaraj S. Myeloid-derived suppressor cells as regulators of the immune system. Nat Rev Immunol. 2009;9: $162-74$.

46. Merad M, Sathe P, Helft J, et al. The dendritic cell lineage: ontogeny and function of dendritic cells and their subsets in the steady state and the inflamed setting. Annu Rev Immunol. 2013; 31:563-604.

47. McKenna K, Beignon AS, Bhardwaj N. Plasmacytoid dendritic cells: linking innate and adaptive immunity. J Virol. 2005;79: 17-27.

48. Kolaczkowska E, Kubes P. Neutrophil recruitment and function in health and inflammation. Nat Rev Immunol. 2013;13:159-75.

49. Stein JV, Nombela-Arrieta C. Chemokine control of lymphocyte trafficking: a general overview. Immunology. 2005;116:1-12.

50. Ngiow SF, Young A, Jacquelot N, et al. A threshold level of intratumor CD8+ T-cell PD1 expression dictates therapeutic response to anti-PD1. Cancer Res. 2015;75:3800-11.

51. Kamphorst AO, Pillai RN, Yang S, et al. Proliferation of PD-1+ CD8 T cells in peripheral blood after PD-1-targeted therapy in lung cancer patients. Proc Natl Acad Sci USA. 2017;114:4993-8.

52. Hackstein H, Wachtendorf A, Kranz S, et al. Heterogeneity of respiratory dendritic cell subsets and lymphocyte populations in inbred mouse strains. Respir Res. 2012;13:94.

53. Velasquez-Lopera MM, Correa LA, Garcia LF. Human spleen contains different subsets of dendritic cells and regulatory $\mathrm{T}$ lymphocytes. Clin Exp Immunol. 2008;154:107-14.

54. Langeveld M, Gamadia LE, ten Berge IJ. T-lymphocyte subset distribution in human spleen. Eur J Clin Invest. 2006;36:250-6. 
55. Asselin-Paturel C, Brizard G, Pin JJ, et al. Mouse strain differences in plasmacytoid dendritic cell frequency and function revealed by a novel monoclonal antibody. J Immunol. 2003;171: 6466-77.

56. Watanabe $\mathrm{H}$, Numata $\mathrm{K}$, Ito $\mathrm{T}$, et al. Innate immune response in Th1- and Th2-dominant mouse strains. Shock. 2004; 22:460-6.

57. Rivera J, Tessarollo L. Genetic background and the dilemma of translating mouse studies to humans. Immunity. 2008;28:1-4.

58. Gueders MM, Paulissen G, Crahay C, et al. Mouse models of asthma: a comparison between $\mathrm{C} 57 \mathrm{BL} / 6$ and $\mathrm{BALB} / \mathrm{c}$ strains regarding bronchial responsiveness, inflammation, and cytokine production. Inflamm Res. 2009;58:845-54.

59. Schulte S, Sukhova GK, Libby P. Genetically programmed biases in Th1 and Th2 immune responses modulate atherogenesis. Am J Pathol. 2008;172:1500-8.

60. Lechner MG, Karimi SS, Barry-Holson K, et al. Immunogenicity of murine solid tumor models as a defining feature of in vivo behavior and response to immunotherapy. J Immunother. 2013; 36:477-89.

61. Jovicic N, Jeftic I, Jovanovic I, et al. Differential immunometabolic phenotype in Th1 and Th2 dominant mouse strains in response to high-fat feeding. PLoS ONE. 2015;10:e134089.

62. Florez-Vargas O, Brass A, Karystianis G, et al. Bias in the reporting of sex and age in biomedical research on mouse models. eLife. 2016;5:e13615.

63. Ito C, Okuyama-Dobashi K, Miyasaka T, et al. CD8+ T cells mediate female-dominant IL-4 production and airway inflammation in allergic asthma. PLoS ONE. 2015;10:e0140808.

64. Lloyd CM, Hawrylowicz CM. Regulatory $\mathrm{T}$ cells in asthma. Immunity. 2009;31:438-49.

65. Rothermel AL, Gilbert KM, Weigle WO. Differential abilities of Th1 and Th2 to induce polyclonal B cell proliferation. Cell Immunol. 1991;135:1-15.

66. Bryan MA, Guyach SE, Norris KA. Specific humoral immunity versus polyclonal B cell activation in Trypanosoma cruzi infection of susceptible and resistant mice. PLoS Negl Trop Dis. 2010;4: e733.

67. Morbach H, Eichhorn EM, Liese JG, et al. Reference values for B cell subpopulations from infancy to adulthood. Clin Exp Immunol. 2010;162:271-9.

68. Pyzik M, Kielczewska A, Vidal SM. NK cell receptors and their MHC class I ligands in host response to cytomegalovirus: insights from the mouse genome. Semin Immunol. 2008;20: $331-42$.

69. Lee SH, Girard S, Macina D, et al. Susceptibility to mouse cytomegalovirus is associated with deletion of an activating natural killer cell receptor of the C-type lectin superfamily. Nat Genet. 2001;28:42-45.

70. Ouzounova M, Lee E, Piranlioglu R, et al. Monocytic and granulocytic myeloid derived suppressor cells differentially regulate spatiotemporal tumour plasticity during metastatic cascade. Nat Commun. 2017;8:14979.

71. Ortiz ML, Kumar V, Martner A, et al. Immature myeloid cells directly contribute to skin tumor development by recruiting IL-17producing CD4+ T cells. J Exp Med. 2015;212:351-67.

72. Schmid M, Zimara N, Wege AK, et al. Myeloid-derived suppressor cell functionality and interaction with Leishmania major parasites differ in C57BL/6 and BALB/c mice. Eur J Immunol. 2014;44:3295-306.

73. Van Ginderachter JA, Beschin A, De Baetselier P, et al. Myeloidderived suppressor cells in parasitic infections. Eur J Immunol. 2010;40:2976-85.

74. Santos JL, Andrade AA, Dias AA, et al. Differential sensitivity of C57BL/6 (M-1) and BALB/c (M-2) macrophages to the stimuli of IFN-gamma/LPS for the production of NO: correlation with iNOS mRNA and protein expression. J Interferon Cytokine Res. 2006;26:682-8.

75. Depke M, Breitbach K, Dinh Hoang Dang K, et al. Bone marrowderived macrophages from $\mathrm{BALB} / \mathrm{c}$ and $\mathrm{C} 57 \mathrm{BL} / 6$ mice fundamentally differ in their respiratory chain complex proteins, lysosomal enzymes and components of antioxidant stress systems. J Proteomics. 2014;103:72-86.

76. Bertolini TB, de Souza AI, Gembre AF, et al. Genetic background affects the expansion of macrophage subsets in the lungs of Mycobacterium tuberculosis-infected hosts. Immunology. 2016; 148:102-13.

77. Billard E, Cazevieille C, Dornand J, et al. High susceptibility of human dendritic cells to invasion by the intracellular pathogens Brucella suis, B. abortus, and B. melitensis. Infect Immun. 2005; 73:8418-24.

78. Pina A, de Araujo EF, Felonato M, et al. Myeloid dendritic cells (DCs) of mice susceptible to paracoccidioidomycosis suppress $\mathrm{T}$ cell responses whereas myeloid and plasmacytoid DCs from resistant mice induce effector and regulatory $\mathrm{T}$ cells. Infect Immun. 2013;81:1064-77.

79. Lande R, Gilliet M. Plasmacytoid dendritic cells: key players in the initiation and regulation of immune responses. Ann NY Acad Sci. 2010;1183:89-103.

80. Zhang H, Gregorio JD, Iwahori T, et al. A distinct subset of plasmacytoid dendritic cells induces activation and differentiation of $\mathrm{B}$ and T lymphocytes. Proc Natl Acad Sci USA. 2017;114:1988-93.

81. Sousa LM, Carneiro MB, Resende ME, et al. Neutrophils have a protective role during early stages of Leishmania amazonensis infection in BALB/c mice. Parasite Immunol. 2014;36:13-31.

82. Chen L, Watanabe T, Watanabe H, et al. Neutrophil depletion exacerbates experimental Chagas' disease in BALB/c, but protects C57BL/6 mice through modulating the Th1/Th2 dichotomy in different directions. Eur J Immunol. 2001;31:265-75.

83. Leynaert B, Sunyer J, Garcia-Esteban R, et al. Gender differences in prevalence, diagnosis and incidence of allergic and non-allergic asthma: a population-based cohort. Thorax. 2012;67:625-31. 